\title{
OPTICAL VORTICES IN DISPERSIVE NONLINEAR KERR-TYPE MEDIA
}

\author{
LUBOMIR M. KOVACHEV
}

Received 2 January 2003

\begin{abstract}
The applied method of slowly varying amplitudes gives us the possibility to reduce the nonlinear vector integrodifferential wave equation of the electrical and magnetic vector fields to the amplitude vector nonlinear differential equations. Using this approximation, different orders of dispersion of the linear and nonlinear susceptibility can be estimated. Critical values of parameters to observe different linear and nonlinear effects are determined. The obtained amplitude equations are a vector version of 3D +1 nonlinear Schrödinger equation (VNSE) describing the evolution of slowly varying amplitudes of electrical and magnetic fields in dispersive nonlinear Kerr-type media. We show that VNSE admits exact vortex solutions with classical orbital momentum $\ell=1$ and finite energy. Dispersion region and medium parameters necessary for experimental observation of these vortices are determined.
\end{abstract}

2000 Mathematics Subject Classification: 35Q55, 45G15, 90C30.

1. Introduction. At the present time, there are no difficulties for experimentalist in laser physics and nonlinear optics to obtain picosecond or femtosecond optical pulses with equal durations in $x, y$, and $z$ directions. The problems with the so-generated light bullets arise in the process of their propagation in a nonlinear media with dispersion. In the transparency region of a dispersive Kerr-type media, as it was established in [6, 14], the scalar paraxial approximation (no dispersion in the direction of propagation) is in a very good accordance with the experimental results. The paraxial approximation, used in the derivation of the scalar 2D +1 nonlinear Schrödinger equation (NLS), does not include (in first- or second-order of magnitude) a small second derivative of the amplitude function in the direction of propagation. The inclusion of this term does not change the main result dramatically in the case of a linear propagation (pulses with small intensity), as generated optical bullets at short distance are transformed in optical disks, with large transverse and small lengthwise dimension. Some of the experimental possibilities for this small term to become important were discussed in [21]. In [2] it was shown that this second derivative in the direction of propagation term is in the same order and with the same sign as the others only in some special cases: optical pulses near the Langmuir frequency or near some of the electronic resonances. In these regions, the sign of the dispersion is negative and the scalar $2 \mathrm{D}+1$ NLS becomes a 3D +1 NLS one. Propagation of optical bullets under the dynamics of $2 \mathrm{D}+1$ and $3 \mathrm{D}+1$ NLS is investigated also in relation to different kinds of nonlinearity [9, 10, 19, 23, 24]. A generation of a new kind of 2D and 3D optical pulses, the so-called optical vortices, has recently become a topic of considerable interest. Generally, the optical vortices 
are such type of optical pulses which admit angular dependence of electrical field or helical phase distribution. The electrical field or intensity is zero also in the center of the vortices. The original scalar theory of optical vortices was based on the wellknown 2D +1 NLS $[7,25,26]$. In a self-focusing regime of propagation, optical rings can be generated, but they are modulationally unstable [18, 27]. One alternative way of stabilizing optical vortices in 2D and 3D cases, using saturable $[11,28]$ or cubicquintic $[8,17]$ nonlinearity, was discussed also. On the other hand, the experiments with optical vortices show that the polarization and the vector character of the electric field play an important role in the dynamics and the stabilization of the vortices [16]. To investigate these cases, we are going to a vector version of 3D +1 NLS. In Section 2, we derive this vector version of 3D +1 NLS (VNSE), using also dispersion in the direction of propagation and envelope approximation in the standard way as it was constructed in $[13,20]$. This vector generalization allows us to find exact vortex solutions with spin $l=1$ and finite energy. The question for shape and finiteness energy of these vortices is crucial and will be discussed extensively in Section 5 .

2. Maxwell's equations for a source-free, dispersive, nonlinear Kerr-type medium. The Maxwell's equations for a source-free dispersive medium with Kerr nonlinearity are

$$
\begin{gathered}
\nabla \times \vec{E}=-\frac{1}{c} \frac{\partial \vec{B}}{\partial t}, \\
\nabla \times \vec{H}=\frac{1}{c} \frac{\partial \vec{D}}{\partial t}, \\
\nabla \cdot \vec{D}=0, \\
\nabla \cdot \vec{B}=\nabla \cdot \vec{H}=0, \\
\vec{B}=\vec{H}, \quad \vec{D}=\vec{P}_{\text {lin }}+4 \pi \vec{P}_{\text {nlin }},
\end{gathered}
$$

where $\vec{E}$ and $\vec{H}$ are the electric and magnetic intensity fields, $\vec{D}$ and $\vec{B}$ are the electric and magnetic induction fields, and $\vec{P}_{\text {lin }}$ and $\vec{P}_{\text {nlin }}$ are the linear and nonlinear polarizations of the medium, respectively. The linear electric polarization of an isotropic, disperse medium can be represented as

$$
\begin{aligned}
\vec{P}_{\text {lin }} & =\int_{-\infty}^{t}\left(\delta(t-\tau)+4 \pi \chi^{(1)}(t-\tau)\right) \vec{E}(\tau, x, y, z) d \tau \\
& =\int_{-\infty}^{t} \varepsilon_{0}(t-\tau) \vec{E}(\tau, x, y, z) d \tau,
\end{aligned}
$$

where $\chi^{(1)}$ and $\varepsilon_{0}$ are the linear electric susceptibility and the dielectric constant. In the following, we study such a polarization (linearly or circularly polarized light), where the nonlinear polarization in the case of one carrying frequency can be expressed as

$$
\begin{aligned}
\vec{P}_{n l}^{(3)}=\int_{-\infty}^{t} \int_{-\infty}^{t} \int_{-\infty}^{t} & x^{(3)}\left(t-\tau_{1}, t-\tau_{2}, t-\tau_{3}\right) \\
& \times\left(\vec{E}\left(\tau_{1}, x, y, z\right) \cdot \vec{E}^{*}\left(\tau_{2}, x, y, z\right)\right) \vec{E}\left(\tau_{3}, x, y, z\right) d \tau_{1} d \tau_{2} d \tau_{3} .
\end{aligned}
$$


It is very important to point out here the remark of Akhmanov et al. [1] that the method slowly varying amplitudes of electrical and magnetic fields applied to such nonstationary linear and nonlinear representations is valid when the optical pulse duration of the pulses $t_{0}$ is greater than the characteristic response time of the media $\tau_{0}\left(t_{0} \gg \tau_{0}\right)$, as well as when the time duration of the pulses is less than or equal to the time response of the media $\left(t_{0} \leq \tau_{0}\right)$. This possibility is discussed in the process of deriving the amplitude equations.

Taking the curl of (2.1) and using (2.2) and (2.5), we then obtain

$$
\Delta \vec{E}-\nabla(\nabla \cdot \vec{E})-\frac{1}{c^{2}} \frac{\partial^{2} \vec{D}}{\partial t^{2}}=0
$$

where $\Delta \equiv \nabla^{2}$ is the Laplacian operator. Equation (2.8) is derived without the use of the third Maxwell's equation. Using (2.3) and the expressions for the linear and nonlinear polarizations (2.6) and (2.7), the second term in (2.8) for arbitrary localized vector function of the electrical field can be estimated. As shown in [2], for such type of functions, $\nabla \cdot \vec{E} \cong 0$ and (2.8) is written as

$$
\Delta \vec{E}-\frac{1}{c^{2}} \frac{\partial^{2} \vec{D}}{\partial t^{2}}=0
$$

We define a complex presentation of the real electrical field by the relation

$$
\vec{E}(x, y, z, t)=\vec{A}^{\prime}(x, y, z, t) \frac{1}{2 i}\left(\exp \left(i\left(k_{0} z-\omega_{0} t\right)\right)-c \cdot c\right)
$$

where $\vec{A}^{\prime}, \omega_{0}$, and $k_{0}$ are the real vector amplitude, the optical frequency, and the wave number of the optical field, respectively. The real vector amplitude $\vec{A}^{\prime}$ is also represented by a complex vector amplitude field:

$$
\vec{A}^{\prime}(x, y, z, t)=\frac{1}{2 i}\left(\vec{A}(x, y, z, t)-\vec{A}^{*}(x, y, z, t)\right)
$$

where $\vec{A}$ is the complex amplitude of the electrical field. In this way, our real electrical field is presented with four complex fields of the kind

$$
\begin{aligned}
\vec{E}(x, y, z, t)= & -\frac{1}{4}\left(\vec{A} \exp \left(i\left(k_{0} z-\omega_{0} t\right)\right)+\vec{A}^{*} \exp \left(-i\left(k_{0} z-\omega_{0} t\right)\right)\right) \\
& +\frac{1}{4}\left(\vec{A} \exp \left(-i\left(k_{0} z-\omega_{0} t\right)\right)+\vec{A}^{*} \exp \left(i\left(k_{0} z-\omega_{0} t\right)\right)\right) .
\end{aligned}
$$

This special kind of presentation of electrical field is connected with shape and finiteness energy of vortex solutions, and it is discussed in Section 5 . Here we also use the Fourier representations of the complex amplitude function $\vec{A}$ and their time derivatives 
of second order:

$$
\begin{aligned}
\vec{A}(x, y, z, t)=\int_{-\infty}^{+\infty} \vec{A}\left(x, y, z, \omega-\omega_{0}\right) \exp \left(-i\left(\omega_{0}-\omega\right) t\right) d \omega \\
\frac{\partial \vec{A}(x, y, z, t)}{\partial t}=-i \int_{-\infty}^{+\infty}\left(\omega-\omega_{0}\right) \vec{A}\left(x, y, z, \omega-\omega_{0}\right) \\
\quad \times \exp \left(-i\left(\omega-\omega_{0}\right) t\right) d \omega \\
\frac{\partial^{2} \vec{A}(x, y, z, t)}{\partial t^{2}}=-\int_{-\infty}^{+\infty}\left(\omega-\omega_{0}\right)^{2} \vec{A}\left(x, y, z, \omega-\omega_{0}\right) \\
\times \exp \left(i\left(\omega-\omega_{0}\right) t\right) d \omega .
\end{aligned}
$$

The principle of causality requires the next conditions on the response functions:

$$
\begin{gathered}
\varepsilon(t-\tau)=0, \quad X^{(3)}\left(t-\tau_{1}, t-\tau_{2}, t-\tau_{3}\right)=0, \\
t-\tau<0, \quad t-\tau_{i}<0, \quad i=1,2,3 .
\end{gathered}
$$

That is why we can prolong the upper integral boundary to infinity and use standard Fourier presentation [20]:

$$
\begin{aligned}
& \int_{-\infty}^{t} \varepsilon_{0}(\tau-t) \exp (i \omega \tau) d \tau=\int_{-\infty}^{+\infty} \varepsilon_{0}(\tau-t) \exp (i \omega \tau) d \tau, \\
& \int_{-\infty}^{t} \int_{-\infty}^{t} \int_{-\infty}^{t} x^{(3)}\left(t-\tau_{1}, t-\tau_{2}, t-\tau_{3}\right) d \tau_{1} d \tau_{2} d \tau_{3} \\
& \quad=\int_{-\infty}^{+\infty} \int_{-\infty}^{+\infty} \int_{-\infty}^{+\infty} x^{(3)}\left(t-\tau_{1}, t-\tau_{2}, t-\tau_{3}\right) d \tau_{1} d \tau_{2} d \tau_{3} .
\end{aligned}
$$

The spectral presentation of linear optical susceptibility $\hat{\varepsilon}_{0}(\omega)$ is connected to the nonstationary optical response function by the next Fourier transform:

$$
\hat{\varepsilon}_{0}(\omega) \exp (-i \omega t)=\int_{-\infty}^{+\infty} \varepsilon_{0}(t-\tau) \exp (-i \omega \tau) d \tau .
$$

The expression of the spectral presentation of the nonstationary nonlinear optical susceptibility $\hat{\chi}^{(3)}$ is similar:

$$
\begin{aligned}
& \hat{x}^{(3)}(\omega=2 \omega-\omega) \exp (-i \omega t) \\
&=\int_{-\infty}^{+\infty} \int_{-\infty}^{+\infty} \int_{-\infty}^{+\infty} x^{(3)}\left(t-\tau_{1}, t-\tau_{2}, t-\tau_{3}\right) \\
& \quad \times \exp \left(-i\left(\omega\left(\tau_{1}+\tau_{2}+\tau_{3}\right)\right)\right) d \tau_{1} d \tau_{2} d \tau_{3} .
\end{aligned}
$$

From (2.6), (2.10), (2.13), (2.17), and (2.19), for the linear polarization of one of the complex fields $\vec{P}_{\text {lin }}^{\prime}\left[\vec{A} \exp \left(i\left(k_{0} z-\omega t\right)\right)\right]$, we obtain

$$
\begin{aligned}
\vec{P}_{\text {lin }}^{\prime}=\int_{\infty}^{t} \varepsilon_{0}(t-\tau) \exp \left(i\left(k_{0} z-\omega_{0} \tau\right)\right) \int_{-\infty}^{+\infty} \vec{A}\left(x, y, z, \omega-\omega_{0}\right) \\
\\
\times \exp \left(-i\left(\omega-\omega_{0}\right) \tau\right) d \omega d \tau \\
=\exp \left(i k_{0} z\right) \int_{-\infty}^{+\infty} \vec{A}_{i}\left(x, y, z, \omega-\omega_{0}\right) \int_{-\infty}^{+\infty} \varepsilon_{0}(t-\tau) \exp (-i \omega \tau) d \tau d \omega .
\end{aligned}
$$


The second integral in (2.21) is simply (2.19), and the linear polarization can be written in the form

$$
\begin{aligned}
& \vec{P}_{\text {lin }}^{\prime}(x, y, z, t) \\
& \quad=\exp \left(i\left(k_{0} z-\omega_{0} t\right)\right) \int_{-\infty}^{+\infty} \hat{\varepsilon}_{0}(\omega) \vec{A}\left(x, y, z, \omega-\omega_{0}\right) \exp \left(-i\left(\omega-\omega_{0}\right) t\right) d \omega .
\end{aligned}
$$

It is important to point out that similar expressions as (2.22) are also written for the polarization of the complex fields of the kind

$$
\begin{aligned}
& \vec{P}_{\operatorname{lin}}^{\prime}\left[\vec{A} \exp \left(-i\left(k_{0} z-\omega t\right)\right)\right], \\
& \vec{P}_{\operatorname{lin}}^{\prime}\left[\vec{A}^{*} \exp \left(i\left(k_{0} z-\omega t\right)\right)\right], \\
& \vec{P}_{\operatorname{lin}}^{\prime}\left[\vec{A}^{*} \exp \left(-i\left(k_{0} z-\omega t\right)\right)\right] .
\end{aligned}
$$

The above procedures are used also with the nonlinear polarization. When the third harmonic term is neglected, the result is

$$
\begin{aligned}
& \vec{P}^{\text {nlin }}(x, y, z, t) \\
&= \frac{3}{4} \exp \left(i\left(k_{0} z-\omega_{0} t\right)\right) \\
& \quad \times \int_{-\infty}^{+\infty} \hat{\chi}^{(3)}(\omega) \exp \left(-i\left(\omega-\omega_{0}\right) t\right)\left|\vec{A}\left(x, y, z, \omega-\omega_{0}\right)\right|^{2} \vec{A}\left(x, y, z, \omega-\omega_{0}\right) d \omega .
\end{aligned}
$$

Multiplying (2.22) and (2.24) by $1 / c^{2}$, and using the second derivatives in time, we obtain

$$
\begin{aligned}
& \frac{1}{c^{2}} \frac{\partial^{2} \vec{P}_{\operatorname{lin}}^{\prime}(x, y, z, t)}{\partial t^{2}} \\
& =-\exp \left(i\left(k_{0} z-\omega_{0} t\right)\right) \int_{-\infty}^{+\infty} \frac{\omega^{2} \hat{\varepsilon}_{0}(\omega)}{c^{2}} \vec{A}\left(x, y, z, \omega-\omega_{0}\right) \\
& \times \exp \left(-i\left(\omega-\omega_{0}\right) t\right) d \omega \text {, } \\
& \frac{4 \pi}{c^{2}} \frac{\partial^{2} \vec{P}_{\operatorname{nlin}}(x, y, z, t)}{\partial t^{2}} \\
& =-\exp \left(i\left(k_{0} z-\omega_{0} t\right)\right) \int_{-\infty}^{+\infty} \frac{3 \pi \omega^{2} \hat{\chi}^{(3)}(\omega)}{c^{2}}\left|\vec{A}\left(x, y, z, \omega-\omega_{0}\right)\right|^{2} \\
& \times \vec{A}\left(x, y, z, \omega-\omega_{0}\right) \exp \left(-i\left(\omega-\omega_{0}\right) t\right) d \omega .
\end{aligned}
$$

The spectrum of the amplitude function is restricted by writing the wave vectors

$$
k_{\operatorname{lin}}=\sqrt{\frac{\omega^{2} \hat{\varepsilon}_{0}(\omega)}{c^{2}}}, \quad k_{n l}=\sqrt{\frac{3 \pi \omega^{2} \hat{\chi}^{(3)}(\omega)}{c^{2}}}
$$


near the carrying frequency in a Taylor series:

$$
\begin{aligned}
k_{\operatorname{lin}}^{2}(\omega)=\frac{\omega^{2} \hat{\varepsilon_{0}}(\omega)}{c^{2}}= & k^{2}\left(\omega_{0}\right)+\frac{\partial\left(k^{2}\left(\omega_{0}\right)\right)}{\partial \omega_{0}}\left(\omega-\omega_{0}\right) \\
& +\frac{1}{2} \frac{\partial^{2}\left(k^{2}\left(\omega_{0}\right)\right)}{\partial \omega_{0}^{2}}\left(\omega-\omega_{0}\right)^{2}+\cdots, \\
k_{n l}^{2}(\omega)=\frac{\omega^{2} \hat{\chi}^{(3)}(\omega)}{c^{2}}= & k_{n l}^{2}\left(\omega_{0}\right)+\frac{\partial\left(k_{n l}^{2}\left(\omega_{0}\right)\right)}{\partial \omega_{0}}\left(\omega-\omega_{0}\right)+\cdots .
\end{aligned}
$$

It is convenient to express the nonlinear wave vector by linear wave vector and nonlinear refractive index:

$$
k_{n l}^{2}\left(\omega_{0}\right)=\frac{3 \pi \omega_{0}^{2} \hat{\chi}^{(3)}\left(\omega_{0}\right)}{c^{2}}=\frac{\omega_{0}^{2} \hat{\varepsilon}\left(\omega_{0}\right)}{c^{2}} \frac{3 \pi \hat{\chi}^{(3)}\left(\omega_{0}\right)}{\hat{\varepsilon}\left(\omega_{0}\right)}=k_{0}^{2} n_{2},
$$

where

$$
n_{2}\left(\omega_{0}\right)=\frac{3 \pi \hat{\chi}^{(3)}\left(\omega_{0}\right)}{\hat{\varepsilon}\left(\omega_{0}\right)}
$$

is the nonlinear refractive index. We note here that no approximation of the response function is used. There is only one requirement of the spectral presentations (2.19) and (2.20) of the response functions to admit first- and second-order derivatives with respect to frequency (to be smooth functions). So, this method is not limited from the time duration of the response function and we can also apply it when the half-max of the pulses is in order of the time duration of the response function (femtosecond pulses). The restriction is only with respect to the relation between the main frequency $\omega_{0}$ and time duration of the envelope functions $t_{0}$ determined from the relations (2.28) (conditions for slowly varying amplitudes). Putting (2.28) in (2.25) and (2.26), respectively, and keeping in mind the expressions of time derivatives of the amplitude functions (2.14) and (2.15) for the electric field, the second time derivative of the linear polarization of the optical component is represented in the next truncated form:

$$
\begin{aligned}
\frac{1}{c^{2}} & \frac{\partial^{2} \vec{P}_{\text {lin }}^{\prime}(x, y, z, t)}{\partial t^{2}} \\
& =\left(-k_{0}^{2} \vec{A}+\frac{2 i k_{0}}{v} \frac{\partial \vec{A}}{\partial t}+\left(k_{0} k_{0}^{\prime \prime}+\frac{1}{v^{2}}\right) \frac{\partial^{2} \vec{A}}{\partial t^{2}}+\cdots\right) \exp \left(i\left(k_{0} z-\omega_{0} t\right)\right) .
\end{aligned}
$$

A similar result is also obtained for the linear polarization of the terms of kind (2.23). The nonlinear polarization term is

$$
\begin{aligned}
& \frac{4 \pi}{c^{2}} \frac{\partial^{2} \vec{P}_{\text {nlin }}^{\prime}(x, y, z, t)}{\partial t^{2}} \\
& \quad=\left(-k_{0}^{2} n_{2}|\vec{A}|^{2} \vec{A}+2 i k_{n l} \frac{\partial k_{n l}}{\partial \omega} \frac{\partial\left(|\vec{A}|^{2} \vec{A}\right)}{\partial t}+\cdots\right) \exp \left(i\left(k_{0} z-\omega_{0} t\right)\right) \\
& \quad=\left(-k_{0}^{2} n_{2}|\vec{A}|^{2} \vec{A}+\left(2 i k_{0} \frac{n_{2}}{v}+i k_{0}^{2} \frac{\partial n_{2}}{\partial \omega}\right) \frac{\partial\left(|\vec{A}|^{2} \vec{A}\right)}{\partial t}+\cdots\right) \exp \left(i\left(k_{0} z-\omega_{0} t\right)\right),
\end{aligned}
$$


where $v=1 /\left(\partial k_{\operatorname{lin}}\left(\omega_{0}\right) / \partial \omega\right)$ and $k_{0}^{\prime \prime}$ are the group velocity and dispersion. From the wave equation (2.9), using (2.12), (2.31), (2.32), and (2.23), the next two equations of complex vector amplitude and its complex conjugates are obtained:

$$
\begin{aligned}
i\left(\frac{\partial \vec{A}}{\partial t}+\right. & \left.v \frac{\partial \vec{A}}{\partial z}+\left(n_{2}+\frac{k_{0} v}{2} \frac{\partial n_{2}}{\partial \omega}\right) \frac{\partial\left(|\vec{A}|^{2} \vec{A}\right)}{\partial t}\right) \\
& =\frac{v}{2 k_{0}} \Delta \vec{A}-\frac{v}{2}\left(k_{0}^{\prime \prime}+\frac{1}{k_{0} v^{2}}\right) \frac{\partial^{2} \vec{A}}{\partial t^{2}}+\frac{n_{2} k_{0} v}{2}|\vec{A}|^{2} \vec{A}, \\
-i\left(\frac{\partial \vec{A}^{*}}{\partial t}\right. & \left.+v \frac{\partial \vec{A}^{*}}{\partial z}+\left(n_{2}+\frac{k_{0} v}{2} \frac{\partial n_{2}}{\partial \omega}\right) \frac{\partial\left(|\vec{A}|^{2} \vec{A}^{*}\right)}{\partial t}\right) \\
& =\frac{v}{2 k_{0}} \Delta \vec{A}^{*}-\frac{v}{2}\left(k_{0}^{\prime \prime}+\frac{1}{k_{0} v^{2}}\right) \frac{\partial^{2} \vec{A}^{*}}{\partial t^{2}}+\frac{n_{2} k_{0} v}{2}|\vec{A}|^{2} \vec{A}^{*}=0 .
\end{aligned}
$$

Equations (2.33) and (2.34) are equal. Solving (2.33) for $\vec{A}$, we can also find $\vec{A}^{*}$ and, respectively, the real amplitude vector field $\vec{A}^{\prime}$. We investigate the case when

$$
v^{2} k_{0} k_{0}^{\prime \prime}=-1,
$$

a condition that can only be fulfilled for materials possessing negative dispersion. The $\partial^{2} \vec{A} / \partial t^{2}$ term in this case is neglected. Applying a Galilean transformation to the vector amplitude equation (2.33), where the new reference frame moves at the group velocity, $t^{\prime}=t, z^{\prime}=z-v t$ (the primes are missing for clarity), we obtain our final vector amplitude equation

$$
-i\left(\frac{\partial \vec{A}}{\partial t}+\left(n_{2}+\frac{k_{0} v}{2} \frac{\partial n_{2}}{\partial \omega}\right) \frac{\partial\left(|\vec{A}|^{2} \vec{A}\right)}{\partial t}\right)+\frac{v}{2 k_{0}} \Delta_{\perp} \vec{A}-\frac{v^{3} k_{0}^{\prime \prime}}{2} \frac{\partial^{2} \vec{A}}{\partial z^{2}}+\frac{n_{2} k_{0} v}{2}|\vec{A}|^{2} \vec{A}=0
$$

where $\Delta_{\perp}=\partial^{2} / \partial x^{2}+\partial^{2} / \partial y^{2}$.

3. Norming VNSE and exact vortex solutions. In this section, we obtain solutions in the case of amplitude vector equations of one carrying frequency. The case of two carrying frequencies is discussed in [2]. The case of three and more localized optical waves on different frequencies with additional conditions on the frequencies (parametric vortices) will be discussed in a next paper. Defining the rescaled variables

$$
\vec{A}=A_{0} \vec{A}^{\prime}, \quad x=r_{0} x^{\prime}, \quad y=r_{o} y^{\prime}, \quad z=r_{0} z^{\prime}, \quad t=t_{0} t^{\prime},
$$

and constants

$$
\begin{gathered}
\alpha=\frac{2 k_{0} r_{0}^{2}}{t_{0} v}, \quad \beta=v^{2} k^{\prime \prime} k_{0}, \quad \gamma=k_{0}^{2} r_{0}^{2} n_{2}\left|A_{0}\right|^{2}, \\
\frac{r_{0}}{t_{0}}=v, \quad \gamma_{1}=2 k_{0} r_{0} n_{2}\left|A_{0}\right|^{2},
\end{gathered}
$$


(2.36) can be transformed into the following (the primes are not written):

$$
-i\left(\alpha \frac{\partial \vec{A}}{\partial t}+\left(\gamma_{1}+\gamma_{2}\right) \frac{\partial\left(|\vec{A}|^{2} \vec{A}\right)}{\partial t}\right)+\Delta_{\perp} \vec{A}-\beta \frac{\partial^{2} \vec{A}}{\partial z^{2}}+\gamma|\vec{A}|^{2} \vec{A}=0
$$

The dispersion term (the second derivative in $z$ direction of the amplitude function) in the transparency region of a medium is one or two orders of magnitude smaller than the diffractive term (transverse Laplacian). This is why the linear (dispersion) parameter is usually $\beta \sim 10^{-2}$. There is a possibility to reach $\beta=1$ only in some special cases, near to Langmuir frequency in cold plasma, high-frequency transparency region of dielectrics, and near to some of the electronic resonances [2]. The constant $\alpha$ has a value of $\alpha \approx 10^{2}\left(\alpha \approx r_{0} k_{0}\right)$ if the slowly varying approximation is used. When the nonlinear constant admits a typical critical value $\gamma=1$ for self-focusing regime, the constants $\gamma_{1} \cong \gamma_{2} \sim 10^{-2}$ are small. We point out here that the effects of asymmetry of the pulses (asymmetry of their spectrum) due to nonlinear addition to the group velocity presented by the second term in (3.3) are substantial when $\gamma_{1} \cong \gamma_{2} \sim 1$ or $\gamma \sim 10^{2}$. These terms depend on intensity of the fields, and when $\gamma \sim 10^{2}$ or $n_{2}\left|A_{0}\right|^{2} \sim 10^{-2}$, effects of self-steepening of the pulses, connected also with considerable self-focusing, can be estimated. Such a type of experiments [12] is provided with 80 femtosecond pulses and intensity $I_{0} \sim 10^{14} \mathrm{~W} / \mathrm{cm}^{2}$. These correspond to the parameters discussed above $\left(\gamma \sim 10^{2}\right.$ or $n_{2}\left|A_{0}\right|^{2} \sim 10^{-2}$ ). In this paper, we investigate the case when $\beta=-1$ (negative dispersion) and $\gamma=1$. Neglecting the small terms, (3.3) becomes

$$
-i \alpha \frac{\partial \vec{A}}{\partial t}+\Delta_{\perp} \vec{A}+\frac{\partial^{2} \vec{A}}{\partial z^{2}}+|\vec{A}|^{2} \vec{A}=0
$$

Now we go to the next step - the possible polarization of the amplitude vector field (3.4). Recently, this problem was discussed intensively [4, 5] and the difference between the polarization of the optical waves with spectral bandwidth (slowly varying amplitudes) and the polarization of monochromatic fields was pointed out. In the case of monochromatic and quasimonochromatic fields, the Stokes parameters can be constructed from transverse components of the wave field [4]. This leads to two-component vector fields in a plane, transverse to the direction of propagation. For electromagnetic fields with spectral bandwidth (our case), the two-dimensional coherency tensor cannot be used and the Stokes parameters cannot be found directly. As it was shown by Carozzi et al. in [5], using a high order of symmetry (SU(3)), in this case, six independent Stokes parameters can be found. This corresponds to a three-component vector field. We investigate this case here. The increase of the spectral bandwidth of the vector wave increases also the depolarization term (a component normal to the standard Stokes coherent polarization plane). The amplitude vector function of electrical field $\vec{A}$ is represented as the sum of three orthogonal linearly polarized amplitudes:

$$
\vec{A}(x, y, z, t)=\sum_{\vec{j}=\vec{x}, \vec{y}, \vec{z}} \vec{j} A_{j}(x, y, z) \exp (i \Omega t) .
$$


In a Cartesian coordinate system, for solutions of the kind of (3.5), the vector equation is reduced to a scalar system of three nonlinear wave equations:

$$
\alpha \Omega A^{l}+\Delta A^{l}+\sum_{j=x, y, z}\left(\left|A^{j}\right|^{2}\right) A^{l}=0, \quad l=x, y, z
$$

As it was pointed out in [20], we may choose to express each of the components $A_{i}$ in spherical coordinates $A_{i}(r, \theta, \varphi)$ of the independent variables $i=x, y, z$. In this way, the linear unique (polarization) vector of each of the components is kept. The system (3.6) in spherical variables is

$$
\alpha \Omega A^{l}+\Delta_{r} A^{l}+\frac{1}{r^{2}} \Delta_{\theta, \varphi} A^{l}+\sum_{j=x, y, z}\left(\left|A^{j}\right|^{2}\right) A^{l}=0, \quad l=x, y, z
$$

where

$$
\Delta_{r}=\frac{1}{r^{2}} \frac{\partial}{\partial r}\left(r^{2} \frac{\partial}{\partial r}\right), \quad \Delta_{\theta, \varphi}=\frac{1}{\sin \theta} \frac{\partial}{\partial \theta}\left(\sin \theta \frac{\partial}{\partial \theta}\right)+\frac{1}{\sin ^{2} \theta} \frac{\partial^{2}}{\partial \varphi^{2}}
$$

are the radial and the angular operators, respectively, and $r=\sqrt{x^{2}+y^{2}+z^{2}}, \theta=$ $\arccos (z / r)$, and $\varphi=\arctan (x / y)$ are the moving spherical variables of the independent variables $x, y$, and $z$. The system of equations (3.7) is solved using the method of separation of the variables. We present the components of the field as a product of a radial and an angular part:

$$
A^{l}(r, \theta, \varphi)=R(r) Y_{l}(\theta, \varphi), \quad l=x, y, z
$$

with the additional constraint on the angular parts

$$
\left|Y_{x}(\theta, \varphi)\right|^{2}+\left|Y_{y}(\theta, \varphi)\right|^{2}+\left|Y_{z}(\theta, \varphi)\right|^{2}=\text { const }
$$

Multiplying (3.7) by $r^{2} / R Y_{l}$ and bearing in mind the constraint expressed in (3.10), we obtain

$$
\frac{r^{2} \Delta_{r} R}{R}+r^{2}\left(\alpha \Omega+|R|^{2}\right)=-\frac{\Delta_{\theta, \varphi} Y_{l}}{Y_{l}}=\ell(\ell+1),
$$

where $\ell$ is a number. Thus the following equations for the radial and the angular parts of the wave functions are obtained:

$$
\begin{aligned}
& \Delta_{r} R+\alpha \Omega R+|R|^{2} R-\frac{\ell(\ell+1)}{r^{2}} R=0, \\
& \Delta_{\theta, \varphi} Y_{l}+\ell(\ell+1) Y_{l}=0, \quad l=x, y, z .
\end{aligned}
$$

Equations (3.12) and (3.13) show that the nonlinear term occurs only in the radial components of the fields. As for the angular parts, we have the usual linear eigenvalue 
problem. The solutions of (3.13) of the angular parts are well known; each of them has exact solutions of the form

$$
Y_{l}=Y_{\ell}^{m}=\Theta_{\ell}^{m}(\theta) \Phi_{m}(\varphi)=\sqrt{\frac{4 \pi}{3}} \sqrt{\frac{2 \ell+(\ell-m) !}{4 \pi(\ell+m) !}} P_{\ell}^{m}(\cos \theta) \exp (\text { im } \varphi),
$$

where $P_{\ell}^{m}$ are the Legendre functions for a discrete series of numbers: $\ell=0,1,2, \ldots$, $m=0, \pm 1, \pm 2, \ldots$, and $|m|<\ell$. Returning to (3.7), it is seen that separation of variables for spherical functions, which satisfy condition (3.10), is possible only for $l=1$ :

$$
Y_{x}=Y_{1}^{-1}=\sin \theta \cos \varphi, \quad Y_{y}=Y_{1}^{1}=\sin \theta c \sin \varphi, \quad Y_{z}=Y_{1}^{0}=\cos \theta,
$$

or another appropriate combination of axes. By choosing one of these angular components for each of the field components, we see that the eigenfunctions (3.15) are solutions to the angular part of the set of equations (3.13). It is straightforward to show that the radial part of (3.12) admits "de Broglie soliton" solutions [3] of the form

$$
R=\sqrt{2} \frac{\exp (i \sqrt{\alpha \Omega} r)}{r} .
$$

In this way, we prove the existence of vortex solutions with angular momentum $l=1$ in a Kerr-type medium. The solutions of the vector amplitude equation (3.4) in a fixed basis are

$$
\begin{aligned}
& A_{x}=\sqrt{2} \frac{\exp (i \sqrt{\alpha \Omega} r)}{r} \sin \theta \cos \varphi \exp (i \Omega t) \\
& A_{y}=\sqrt{2} \frac{\exp (i \sqrt{\alpha \Omega} r)}{r} \sin \theta \sin \varphi \exp (i \Omega t) \\
& A_{z}=\sqrt{2} \frac{\exp (i \sqrt{\alpha \Omega} r)}{r} \cos \theta \exp (i \Omega t)
\end{aligned}
$$

Equation (3.4) is normed and this leads to the following normed constants:

$$
\alpha \Omega=1, \quad \Omega \cong 10^{-2} .
$$

Now we can turn back to the amplitude of real solutions $\vec{A}^{\prime}$, using (2.11),

$$
\begin{aligned}
& A_{x}^{\prime}=\frac{1}{2 i}\left(A_{x}-A_{x}^{*}\right)=\sqrt{2} \frac{\sin (\sqrt{\alpha \Omega} r+\Omega t)}{r} \sin \theta \cos \varphi, \\
& A_{y}^{\prime}=\frac{1}{2 i}\left(A_{y}-A_{y}^{*}\right)=\sqrt{2} \frac{\sin (\sqrt{\alpha \Omega} r+\Omega t)}{r} \sin \theta \sin \varphi, \\
& A_{z}^{\prime}=\frac{1}{2 i}\left(A_{z}-A_{z}^{*}\right)=\sqrt{2} \frac{\sin (\sqrt{\alpha \Omega} r+\Omega t)}{r} \cos \theta .
\end{aligned}
$$

The condition $\nabla \cdot \vec{E}=0$ splits the nonlinear Maxwell system into two wave equation systems, one nonlinear of the electrical field, and the other nonlinear of the magnetic field. We define again a complex presentation of the real magnetic field by the relation

$$
\vec{H}(x, y, z, t)=\vec{C}^{\prime}(x, y, z, t) \frac{1}{2 i}\left(\exp \left(i\left(k_{0} z-\omega_{0} t\right)\right)-c \cdot c\right),
$$


where $\vec{C}^{\prime}, \omega_{0}$, and $k_{0}$ are the real vector amplitude, the optical frequency, and the wave number of the optical field, respectively. The real magnetic vector amplitude $\vec{C}^{\prime}$ is represented also by a complex vector amplitude field:

$$
\vec{C}^{\prime}(x, y, z, t)=\frac{1}{2 i}\left(\vec{C}(x, y, z, t)-\vec{C}^{*}(x, y, z, t)\right),
$$

where $\vec{C}$ is the complex amplitude of the magnetic field. Applying similar procedures to those which were made for the electrical field, namely, using the Fourier representation of the amplitude functions $\vec{C}$ and their time derivatives of second order, as was done for the electrical field, we obtain the vector equation of slowly varying amplitudes of the magnetic field $\vec{C}$ :

$$
i \alpha \frac{\partial \vec{C}}{\partial t}+\Delta_{\perp} \vec{C}+\frac{\partial^{2} \vec{C}}{\partial z^{2}}+|\vec{A}|^{2} \vec{C}=0
$$

if the condition

$$
\nabla|\vec{A}|^{2} \times \vec{A}=0
$$

is satisfied. For solutions of the amplitudes of electrical field like (3.17), (3.18), and (3.19), condition (3.27) is satisfied. Equation (3.26) has a localized solution of the same kind of (3.17), (3.18), and (3.19), as the amplitude functions of the electrical field, but with opposite phase:

$$
\begin{aligned}
& C_{x}=\sqrt{2} \frac{\exp (i \sqrt{\alpha \Omega} r)}{r} \sin \theta \cos \varphi \exp (-i \Omega t), \\
& C_{y}=\sqrt{2} \frac{\exp (i \sqrt{\alpha \Omega} r)}{r} \sin \theta \sin \varphi \exp (-i \Omega t), \\
& C_{z}=\sqrt{2} \frac{\exp (i \sqrt{\alpha \Omega} r)}{r} \cos \theta \exp (-i \Omega t) .
\end{aligned}
$$

The real dynamics of localized fields can be understood only by investigating both (3.3) and (3.26).The solutions of electrical (3.17), (3.18), and (3.19) and magnetic (3.28) fields are with opposite phases, and that is why the corresponding electromagnetic localized wave oscillating in coordinate system moves with group velocity.

4. Experimental conditions. The results presented above require the satisfaction of the conditions relating the linear parameter $\beta$ and the nonlinear parameter $\gamma$; namely, $\beta=-1$ and $\gamma=1$. The relation $\gamma=1$ is a typical critical value for starting a self-focusing regime of a Gaussian pulse. The nonlinear parameter written again is

$$
\gamma=r_{0}^{2} k_{0}^{2} n_{2}\left|A_{0}\right|^{2}=\alpha^{2} n_{2}\left|A_{0}\right|^{2}=1 .
$$

The constant $\alpha=r_{0} k_{i}$ in the optical region ranges in values from $10^{2}$ to $10^{3}$. Using this range in the condition (4.1), we obtain a required nonlinear refractive index change:

$$
n_{2}\left|A_{0}\right|^{2} \cong 10^{-4}-10^{-6} \text {. }
$$


It was shown, using relations (3.2), that if $\gamma=1$, then $\gamma_{1} \sim \gamma_{2} \sim 10^{-2}-10^{-3}$. This is one important result: the nonlinear addition to the group velocity term in amplitude equation (3.3) is relatively small, even for (femtosecond) pulses with time duration less than or equal to characteristic time response of the nonlinear medium.

Using the condition of linear parameter $\beta$,

$$
\beta=k_{0} v^{2} k^{\prime \prime}=-1,
$$

we see that the vortex propagation takes place only in the negative dispersion region. In [2], we find that the constraint given in (4.3) corresponds to the next two experimental situations:

(1) cold plasma near the Langmuir frequency,

(2) a region near an electronic resonance in an isotropic medium.

We point out also the equivalence in high-frequency region between the spectral presentation of dielectric susceptibility of a cold plasma and this of a dielectric medium. The expression is supposed to be precisely equal to a (dielectric) constant. Near these frequencies, the dispersion parameter increases rapidly and admits values

$$
\left|k^{\prime \prime}\right| \sim 10^{-24}-10^{-25} \sec ^{2} / \mathrm{cm} \text {. }
$$

This leads to the fact that the dispersion term (the second derivative in $z$ direction of the amplitude function) in normed amplitude equations of electrical (3.3) and magnetic (3.26) fields is of the same order of the diffractive term (transverse Laplacian).

5. Finiteness of the energy of the vortex solutions. Now we come to the crucial point-energy and shape of the vortex solutions (3.21), (3.22), (3.23), and (3.28) of the electrical and magnetic fields. To prove the finiteness of energy of the vortex solutions (3.21), (3.22), (3.23), and (3.28), we start with the equations for average time balance of energy density of electrical and magnetic fields [15]:

$$
\frac{\partial W}{\partial t}=\frac{1}{16 \pi}\left(\vec{E} \cdot \frac{\partial \vec{D}^{*}}{\partial t}+\vec{E}^{*} \cdot \frac{\partial \vec{D}}{\partial t}+\vec{B} \cdot \frac{\partial \vec{B}^{*}}{\partial t}+\vec{B}^{*} \cdot \frac{\partial \vec{B}}{\partial t}\right),
$$

where $\vec{D}=\vec{P}_{\text {lin }}+4 \pi \vec{P}_{\text {nlin }}$ is the sum of the linear induction and the nonlinear induction of the electrical field. In many books, the calculations of the averaged energy of the optical waves in a dispersive medium are worked out bearing in mind only the first order of slowly varying amplitude approximation of the linear electrical induction. This is why the result comes to the old result of Brillouin (1921) for the energy density of electrical field:

$$
W_{\text {lin }}=\frac{1}{8 \pi}\left(\frac{\partial\left(\omega \epsilon_{0}\right)}{\partial \omega}|\vec{A}|^{2}+|\vec{C}|^{2}\right)=\frac{1}{8 \pi}\left(\frac{c}{v}|\vec{A}|^{2}+|\vec{C}|^{2}\right)
$$

It is straightforward to show using this approximation that after integrating in space our vortex solutions, they admit infinite energy. As seen from (5.2), this approximation does not include the dispersion parameter $k^{\prime \prime}$, which is present in the envelope (3.3) and (3.26) as a coefficient in front of the second derivative in $z$ direction. So there is 
no possibility to estimate the influence of $k^{\prime \prime}$ in the energy integral. Our vector amplitude equations are obtained using the second-order approximation of $\vec{P}_{\text {lin }}$. The right expression of the energy density in this case requires expanding the linear electrical inductions in the energy integral also up to the second order and including this dispersion parameter in the energy integral. Using truncated form for first derivative in time of the linear induction of electrical field, we have

$$
\frac{\partial \vec{P}_{\mathrm{lin}}}{\partial t}=-i \omega_{0} \epsilon\left(\omega_{0}\right) \vec{A}+\frac{c}{v} \frac{\partial \vec{A}}{\partial t}+\frac{i c k^{\prime \prime}}{2} \frac{\partial^{2} \vec{A}}{\partial t^{2}} .
$$

From (5.1), (5.3), and the complex conjugate of (5.3), we obtain

$$
\frac{\partial W_{\text {lin }}}{\partial t}=\frac{c}{v}\left(\vec{A} \frac{\partial \vec{A}^{*}}{\partial t}+\vec{A}^{*} \frac{\partial \vec{A}}{\partial t}\right)+\frac{i c k^{\prime \prime}}{2} \frac{\partial}{\partial t}\left(\vec{A}^{*} \frac{\partial \vec{A}}{\partial t}-\vec{A} \frac{\partial \vec{A}^{*}}{\partial t}\right)+\vec{B} \frac{\partial \vec{B}^{*}}{\partial t}+\vec{B}^{*} \frac{\partial \vec{B}}{\partial t}
$$

or

$$
W_{\text {lin }}=\frac{c}{v}|\vec{A}|^{2}+|\vec{C}|^{2}+\frac{i c k^{\prime \prime}}{2}\left(\vec{A}^{*} \frac{\partial \vec{A}}{\partial t}-\vec{A} \frac{\partial \vec{A}^{*}}{\partial t}\right)
$$

where $A^{*}$ denotes the complex conjugate in time amplitude function. Rewriting the vortex solutions of electrical (3.21), (3.22), and (3.23) and magnetic (3.28) fields in unnormed (dimension) coordinates and substituting in (5.5), for the case of negative dispersion, we finally obtain the next expression of the linear part of averaged energy density:

$$
W_{\text {lin }}=\left(1+\frac{c}{v}-c\left|k^{\prime \prime}\right| \triangle \omega\right)|\vec{A}|^{2},
$$

where $\triangle \omega$ denotes the spectral bandwidth of the vortices. We obtain one unexpected result: with the increase of the spectral bandwidth of our solutions, the linear part of energy density will decrease in the negative dispersion region. Conditions when the linear part of the energy density of electrical field is zero determine one critical spectral bandwidth:

$$
\triangle \omega_{c}=\frac{v+c}{v c} \frac{1}{\left|k^{\prime \prime}\right|}
$$

Near to plasma frequency or some of the electronic resonances, the dispersion parameter increases with values about $k^{\prime \prime} \sim 10^{-24}-10^{-25} \mathrm{cek}^{2} / \mathrm{cm}$. The critical spectral bandwidth in this case becomes $\triangle \omega_{c} \sim 10^{14}-10^{15} \mathrm{~Hz}$. The envelope approximation requires that $\omega_{0}>\triangle \omega_{c}$. This condition shows that the linear part of the energy density of vortices is zero only when their main frequency is situated in optical region, or regions, whose frequency is greater than the optical region frequencies, and admits spectral bandwidth equal to $\triangle \omega_{c}$. The nonlinear part of averaged energy density is expressed in [22], and for the vortex solutions (3.17), (3.18), and (3.19), it becomes

$$
W_{\text {nlin }}=3 n_{2}\left|{\overrightarrow{A^{\prime}}}^{2}{\overrightarrow{A^{\prime}}}^{2}+\omega_{0} \frac{\partial n_{2}}{\partial \omega_{0}}\right|{\overrightarrow{A^{\prime}}}^{2}{\overrightarrow{A^{\prime}}}^{2}
$$


These results give the condition for the finiteness of energy of the vortices that the spectral bandwidth of vortices be equal to $\Delta \omega_{c}$. Integrating $W_{\text {nlin }}$ on the 3D space, we obtain a finite value proportional to the main frequency $\omega_{0}$. The intensity part in this expression for the energy (5.8) is limited by the experimental condition (4.2) and it is a constant. In this way, we obtain the result that the localized energy of the solutions increases linearly with the increase of the main frequency. In addition, we also investigate the shape and the behavior of electrical and magnetic fields in the origin and infinity. To obtain the correct result, the real-part solutions of the amplitude (3.22), (3.23), and (3.28) must be rewritten in the independent Cartesian coordinates $x$, $y$, and $z$ :

$$
\begin{aligned}
& A_{x}^{\prime}=\sqrt{2} \frac{x \sin \left(\sqrt{\alpha \Omega} \sqrt{x^{2}+y^{2}+z^{2}}+\Omega t\right)}{x^{2}+y^{2}+z^{2}}, \\
& A_{y}^{\prime}=\sqrt{2} \frac{y \sin \left(\sqrt{\alpha \Omega} \sqrt{x^{2}+y^{2}+z^{2}}+\Omega t\right)}{x^{2}+y^{2}+z^{2}}, \\
& A_{z}^{\prime}=\sqrt{2} \frac{z \sin \left(\sqrt{\alpha \Omega} \sqrt{x^{2}+y^{2}+z^{2}}+\Omega t\right)}{x^{2}+y^{2}+z^{2}} .
\end{aligned}
$$

The limits of these functions in origin and in infinity are investigated using the Haine criteria for the limit of a multidimensional function. It is straightforward to show that the solutions (5.9) are odd functions, going to zero in infinity and admitting very little value near to the origin. In the origin, using the same criteria, we find that the above functions have no limit. But as we must point out, the envelope functions (5.9) are only imaginary functions around the real electrical field, expressed in our case by relation (2.10):

$$
\begin{aligned}
& E_{x}=A_{x}^{\prime} \sin \left(k_{0} z-\omega_{0} t\right), \\
& E_{y}=A_{y}^{\prime} \sin \left(k_{0} z-\omega_{0} t\right), \\
& E_{z}=A_{z}^{\prime} \sin \left(k_{0} z\right)-\omega_{0} t .
\end{aligned}
$$

Using the same criteria as in the previous case, it is seen that the electrical field admits exact limit zero in the origin and infinity:

$$
\begin{gathered}
\lim _{x, y, z \rightarrow 0}\left(E_{i}\right)=0, \\
\lim _{x, y, z \rightarrow \pm \infty}\left(E_{i}\right)=0, \quad i=x, y, z .
\end{gathered}
$$

6. Numerical experiment. In this section, we compare the evolutions of one-component localized electrical field $A(x, y, z, t)$ whose propagation is governed by the $3 \mathrm{D}+1$ scalar NSE

$$
-i \alpha \frac{\partial A}{\partial t}+\Delta_{\perp} A+\frac{\partial^{2} A}{\partial z^{2}}+|A|^{2} A=0
$$



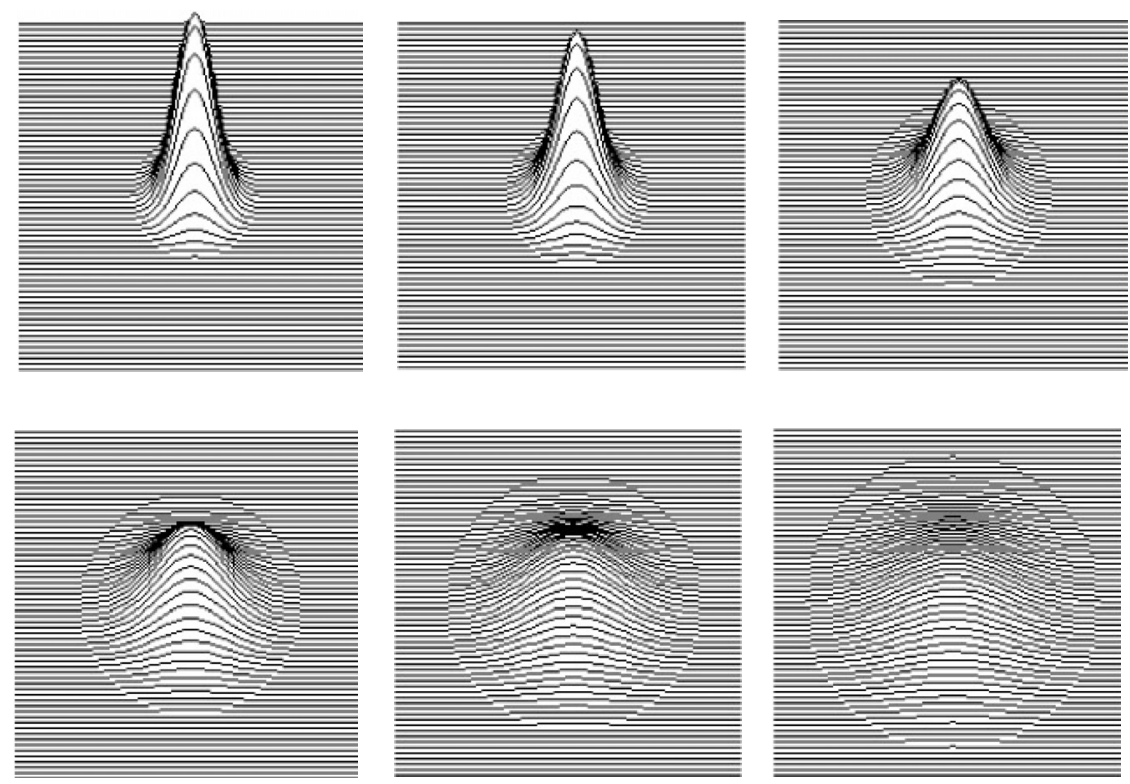

FIGURE 6.1. Evolution of a Gaussian pulse on distance $\pi$ with initial amplitude constant $A_{0}=0.8$, governed by the scalar NSE (6.1). This corresponds to the nonlinear parameter $\gamma<1$ and the dominating diffraction.

with the evolution of vortex solutions (3.17), (3.18), and (3.19) of the vector version of NSE (VNSE)

$$
-i \alpha \frac{\partial \vec{A}}{\partial t}+\Delta_{\perp} \vec{A}+\frac{\partial^{2} \vec{A}}{\partial z^{2}}+|\vec{A}|^{2} \vec{A}=0
$$

We have chosen a split-step Fourier method for solving (6.1) and (6.2).

6.1. Scalar NSE. The amplitude constant $A_{0}=\sqrt{2}$ of the vortex solutions corresponds to a value greater than but close to the critical value for self-focusing of scalar fields $A_{0}=1$ (or $\gamma=1$ ). To compare the results of the scalar theory (6.1) with those of the vector one (6.2), we get the values of amplitude constants in scalar case less than ( $\left.A_{0}=0.8\right)$ or equal $\left(A_{0}=\sqrt{2}\right)$ to the amplitude constant of vortex solutions. The value $A_{0}=0.8$ corresponds to the situation when the diffraction term dominates and the value $A_{0}=\sqrt{2}$ corresponds to a self-focusing regime near to the critical value $\gamma=1$ in dynamics of scalar NSE. The initial condition in the scalar case is Gaussian pulse:

$$
A(x, y, z)=A_{0} \exp \left(-\frac{\sqrt{x^{2}+y^{2}+z^{2}}}{2}\right) .
$$

The evolution of the pulse when $A_{0}=0.8$ governed by scalar NSE (6.1) is presented in Figure 6.1. The upper left graph corresponds to initial condition (6.3) and the lower right graph corresponds to evolution at distance $\pi$. We plotted the surface $A(x, y, z=0, t)$. 

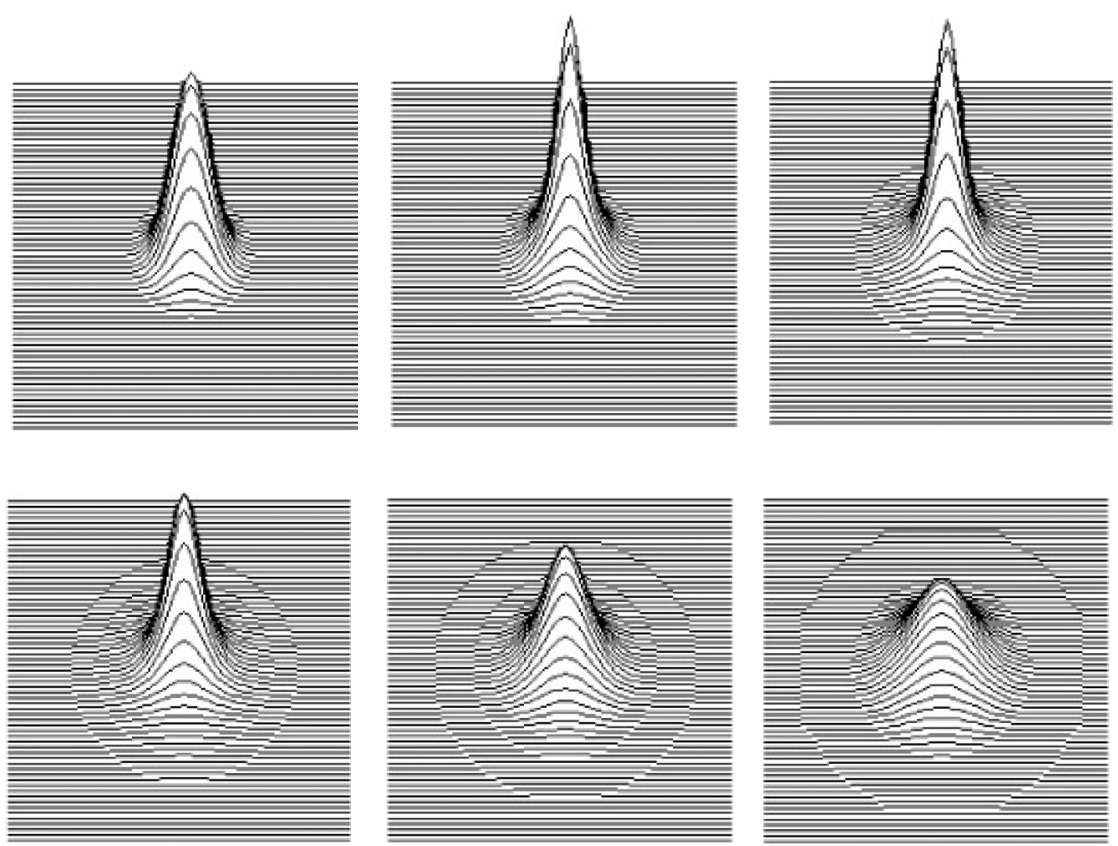

FIGURE 6.2. Evolution of a Gaussian pulse on distance $\pi$ with initial amplitude constant $A_{0}=\sqrt{2}$ (the same as for the vortex solution) governed by the scalar NSE (6.3). The nonlinear parameter $\gamma>1$ is greater than but close to the critical value for self-focusing.

In the same way situated the next two figures also. As it can be expected, the diffractive term dominates and we observe linear (diffractive) regime of propagation. The evolution of Gaussian pulse with $A_{0}=\sqrt{2}$ (equal to vortex initial amplitude) governed by scalar NSE (6.1) is presented in Figure 6.2. The dynamics is close to a real experiment [22]. At the beginning, as the initial constant is greater than but near to the critical value for self-focusing $A_{0}=1$, a self-focusing regime with focusing the central area and forming large base is observed. As the part of intensity distribution goes to the base, the critical power density of the pulse becomes less than the critical power density for self-focusing and again the diffraction dominates.

6.2. Vector NSE. Figure 6.3 presents the result of a numerical experiment of evolution of vortex solutions

$$
\begin{aligned}
& A_{x}=\sqrt{2} \frac{x \sin \left(\sqrt{x^{2}+y^{2}+z^{2}}\right)}{x^{2}+y^{2}+z^{2}}, \\
& A_{y}=\sqrt{2} \frac{y \sin \left(\sqrt{x^{2}+y^{2}+z^{2}}\right)}{x^{2}+y^{2}+z^{2}}, \\
& A_{z}=\sqrt{2} \frac{z \sin \left(\sqrt{x^{2}+y^{2}+z^{2}}\right)}{x^{2}+y^{2}+z^{2}},
\end{aligned}
$$



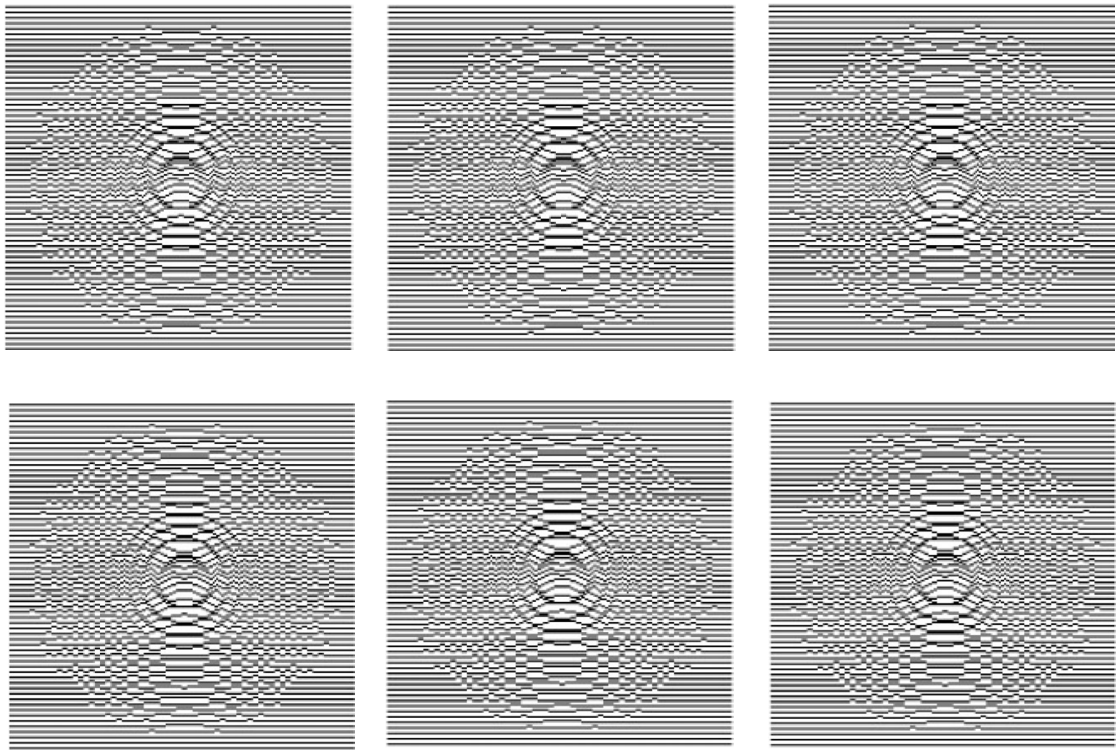

FIGURE 6.3. Evolution of the intensity of the vortex solutions (6.4), (6.5), and (6.6) in dynamics of VNSE (6.2). Stable propagation on distance $\pi$ is established.

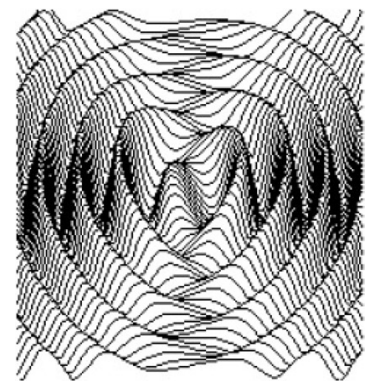

FIGURE 6.4. Surface of one of the components $A_{x}(x, y, z=0)$ for the vortex solution (6.4).

governed by the vector version of NSE (VNSE) (6.2). On the graphs is plotted the dynamics of the surface

$$
|\vec{A}|_{z=0}^{2}=\left|A_{x}\right|_{z=0}^{2}+\left|A_{y}\right|_{z=0}^{2}+\left|A_{z}\right|_{z=0}^{2} .
$$

As it can be established, the vortex is stable on distance $\pi$, where the scalar theory gives an unstable propagation. On Figure 6.4, we present the surface of the solution

$$
A_{x}=\sqrt{2} \frac{x \sin \left(\sqrt{x^{2}+y^{2}+z^{2}}\right)}{x^{2}+y^{2}+z^{2}} \mid z=0 .
$$


The numerical experiment of scalar NSE was performed on an area with grid $128 \times 128 \times$ 128 . The vortex solutions are situated on a large area, and to satisfy zero values on the boundary of the space, we investigate VNSE (6.2) on large space and grid $192 \times 192 \times 192$.

7. Conclusion. The applied method of slowly varying amplitudes gives us the possibility to reduce the nonlinear vector integrodifferential equations of the electrical and magnetic vector fields to vector nonlinear differential equations of amplitudes. Here we explain some more differences between the solutions obtained by separation of the variables of the usual linear scalar Schrödinger equation with potential depending only on " $r$ " (e.g., hydrogen atom) and the solutions of the vector version of NSE. For the linear Schrödinger equations with potential of high order, spherical functions correspond to a high order of radial spherical Bessel functions. While for vector version of NSE, a high number of the fields components and high value of localized energy correspond to a high order of spherical functions $(\ell=1,2, \ldots)$. In the nonlinear case, for radial component, we have for all solutions the zero spherical Bessel function $\sin \alpha r / r$. This is because we start with such a special kind of complexification of our electrical field (2.10). It is important to highlight also the equivalence in high-frequency region between the linear dielectric susceptibility of a cold plasma and that of a dielectric medium. In high-frequency region, we can determine also a plasma frequency of one dielectric. The expression is equal to that of cold plasma and is supposed to be precisely a (dielectric) constant. All this discussion shows two possible regions for observing optical vortices:

(1) high-frequency (transparency) region of cubic dielectrics or cold plasma,

(2) the region near to some of the electronics resonances of an $\chi^{(3)}$ medium.

All the above investigations are provided only for the real part of linear and nonlinear susceptibilities . This is because the first possibility, high-frequency region, is more attractive for observing optical vortices. Near to electronic resonances, the complex part of linear susceptibility is significant and should be kept in mind in a more detailed analysis. The numerical investigation of stability for such type of vortices, using split-step Fourier method, is performed also. Comparing the numerical calculations for vortices with these of a standard Gaussian pulse, it can be clearly seen that the vortices propagate without any changing of their shape, whereas the Gaussian pulse, with the same initial amplitude, is unstable.

\section{REFERENCES}

[1] S. A. Akhmanov, V. A. Vysloukh, and A. S. Chirkin, Optics of Femtosecond Laser Pulses, Nauka, Moscow, 1988 (Russian).

[2] D. R. Andersen and L. M. Kovachev, Interaction of coupled-vector optical vortices, J. Opt. Soc. Amer. B Opt. Phys. 19 (2002), no. 3, 376-384.

[3] A. O. Barut, The Schrödinger and the Dirac equation-linear, nonlinear and integrodifferential, Geometrical and Algebraic Aspects of Nonlinear Field Theory (Amalfi, 1988), North-Holland Delta Ser., North-Holland, Amsterdam, 1989, pp. 37-51.

[4] M. Born and E. Wolf, Principles of Optics, 6th ed., Cambridge University Press, Cambridge, 1998.

[5] T. Carozzi, R. Karlsson, and J. Bergman, Parameters characterizing electromagnetic wave polarization, Phys. Rev. E 61 (2000), no. 2, 2024-2028. 
[6] R. Y. Chiao, E. Garmire, and C. H. Townes, Self-trapping of optical beams, Phys. Rev. Lett. 13 (1964), 479-482.

[7] J. Christou, V. Tikhonenko, Yu. S. Kivshar, and B. Luther-Davies, Vortex soliton motion and steering, Opt. Lett. 21 (1996), no. 20, 1649-1651.

[8] A. Desyatnikov, A. Maimistov, and B. Malomed, Three-dimensional spinning solitons in dispersive media with the cubic-quintic nonlinearity, Phys. Rev. E 61 (2000), no. 3, 31073113.

[9] D. E. Edmundson and R. H. Enns, Fully three-dimensional collisions of bistable light bullets, Opt. Lett. 18 (1993), no. 19, 1609-1611.

[10] _ Particlelike nature of colliding three-dimensional optical solitons, Phys. Rev. A 51 (1995), no. 3, 2491-2498.

[11] W. J. Firth and D. V. Skryabin, Optical solitons carrying orbital angular momentum, Phys. Rev. Lett. 79 (1997), no. 13, 2450-2453.

[12] R. L. Fork, C. V. Shank, C. Hirlimann, R. Yen, and W. J. Tomlinson, Femtosecond white-light continuum pulses, Opt. Lett. 8 (1983), 1-3.

[13] V. I. Karpman, Nonlinear Waves in Dispersive Media, Izdat. Nauka, Moscow, 1973 (Russian).

[14] P. L. Kelley, Self-focusing of optical beams, Phys. Rev. Lett. 15 (1965), 1005-1008.

[15] L. D. Landau and E. M. Lifshitz, Electrodynamics of Continuous Media, Nauka, Moscow, 1978 (Russian).

[16] C. T. Law and G. A. Swartzlander Jr., Polarized optical vortex solitons: instabilities and dynamics in Kerr nonlinear media, Chaos Solitons Fractals 4 (1994), no. 8-9, 17591766.

[17] B. A. Malomed, L.-C. Crasovan, and D. Mihalache, Stability of vortex solitons in the cubicquintic model, Phys. D 161 (2002), no. 3-4, 187-201.

[18] A. V. Mamaev, M. Soffman, and A. A. Zozula, Propagation of dark stripe beams in nonlinear media: snake instability and creation of optical vortices, Phys. Rev. Lett. 76 (1996), no. 13, 2262-2265.

[19] R. McLeod, K. Wagner, and S. Blair, (3+1)-dimensional optical soliton dragging logic, Phys. Rev. A 52 (1995), no. 4, 3254-3278.

[20] A. C. Newell and J. V. Moloney, Nonlinear Optics, Advanced Topics in the Interdisciplinary Mathematical Sciences, Addison-Wesley, California, 1992.

[21] J. A. Powell, J. V. Moloney, A. C. Newell, and R. A. Albanese, Beam collapse as an explanation for anomalous ocular damage, J. Opt. Soc. Amer. B 10 (1993), no. 7, 1230-1241.

[22] Y. R. Shen, The Principles of Nonlinear Optics, John Wiley \& Sons, New York, 1984.

[23] Y. Silberberg, Collapse of optical pulses, Opt. Lett. 15 (1990), no. 22, 1282-1284.

[24] J. M. Soto-Crespo, E. M. Wright, and N. N. Akhmediev, Recurrence and azimuthal-symmetry breaking of a cylindrical Gaussian beam in a saturable self-focusing medium, Phys. Rev. A 45 (1992), no. 5, 3168-3175.

[25] K. Staliunas, Vortices and dark solitons in the two-dimensional nonlinear Schrödinger equation, Chaos Solitons Fractals 4 (1994), no. 8-9, 1783-1796.

[26] G. A. Swartzlander Jr. and C. T. Law, Optical vortex solitons observed in Kerr nonlinear media, Phys. Rev. Lett. 69 (1992), no. 17, 2503-2506.

[27] V. Tikhonenko, J. Christou, and B. Luther-Davies, Spiraling bright spatial solitons formed by the breakup of an optical vortex in a saturable self-focusing medium, J. Opt. Soc. Amer. B 12 (1995), no. 11, 2046-2052.

[28] L. Torner and D. V. Petrov, Azimuthal instabilities and self-breaking of beams into sets of solitons in bulk second-harmonic generation, Electron. Lett. 33 (1997), no. 7, 608-610.

Lubomir M. Kovachev: Institute of Electronics, Bulgarian Academy of Sciences, Tsarigradsko Chaussee 72,1784 Sofia, Bulgaria

E-mail address: 1kovach@i e. bas.bg 


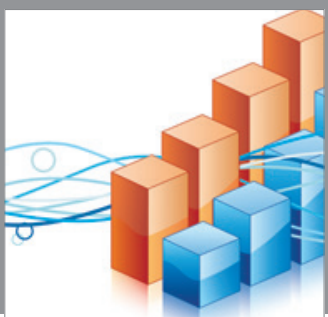

Advances in

Operations Research

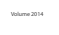

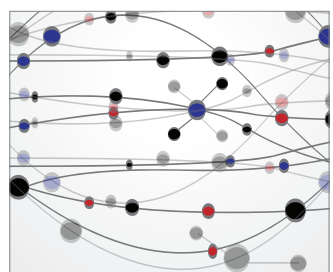

\section{The Scientific} World Journal
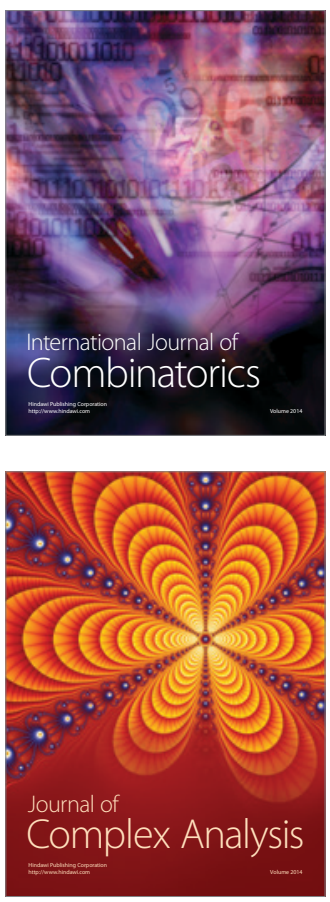

International Journal of

Mathematics and

Mathematical

Sciences
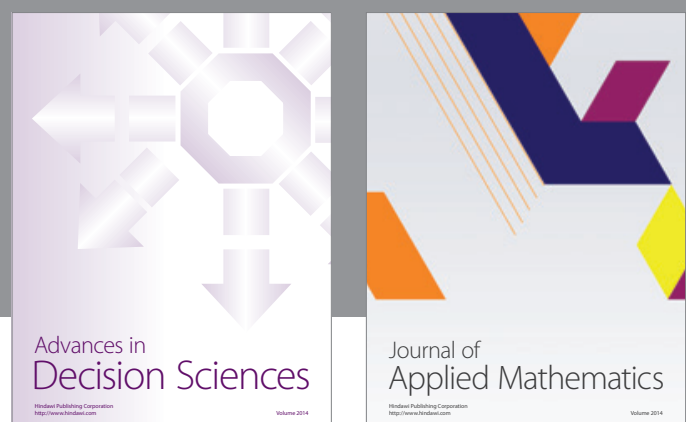

Journal of

Applied Mathematics
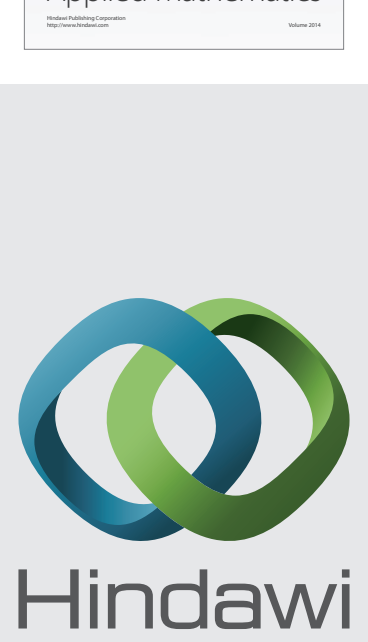

Submit your manuscripts at http://www.hindawi.com
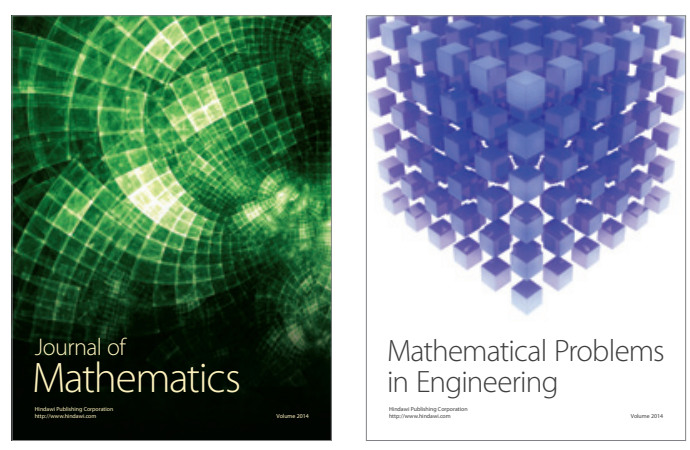

Mathematical Problems in Engineering
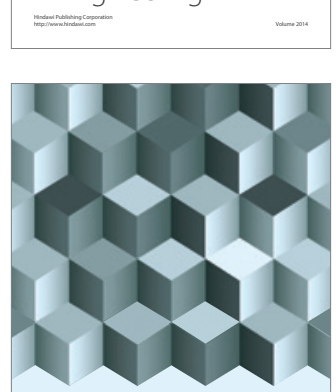

Journal of

Function Spaces
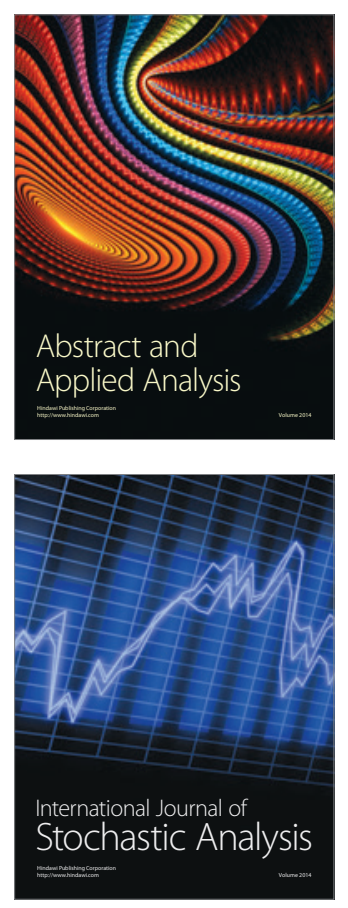

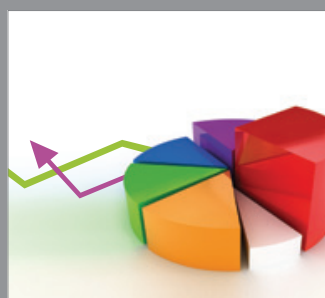

ournal of

Probability and Statistics

Promensencen
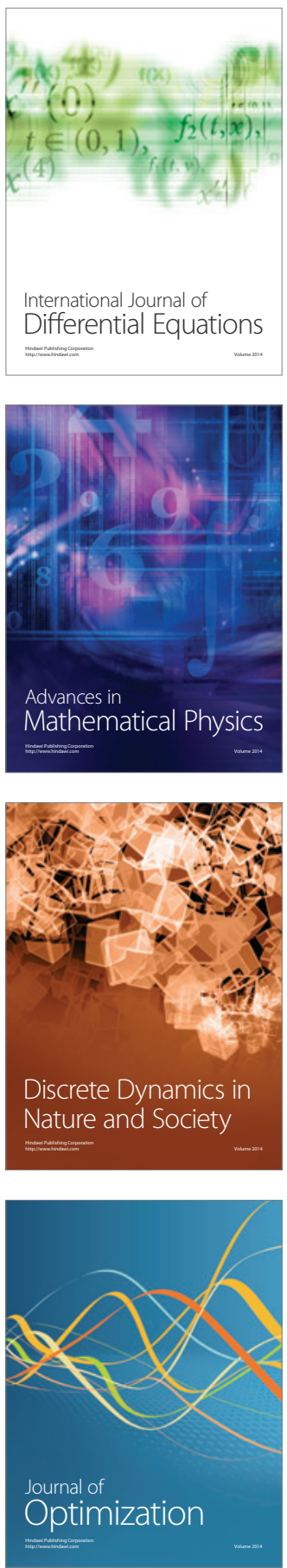